\title{
Flexibility Calculation of Like-U Type Flexure Hinge
}

\author{
Ling Jingjing, Li Ruiqin* and Zhang Qisheng
}

School of Mechanical and Power Engineering North University of China Taiyuan Shanxi 030051, P.R. China

\begin{abstract}
A new like-U type flexure hinge structure is proposed, based on Castigliano's second theorem and calculus theory. Taking the centrifugal angle parameters as the integration variable, and defining the intermediate parameters, it deduces the analytic computational formula for the like-U type flexure hinge flexibility. By changing the structural parameter of the flexure hinge, it is able to transform four different structure flexure hinges, and the deduced analytic computation formula can be applied to all of these four structures flexure hinges. After the twelve flexure hinges of different structures have been analyzed by applying finite-element method, it is found that the results are in good agreement with the results of analytic computation formula. Thus, the validity of the analytic computation formula is verified, realize accurate design and computation for such flexure hinges is realized, and the theoretical base for technical application of like-U type flexure hinge is provided.
\end{abstract}

Keywords: Flexure hinge, flexibility, finite-element method, like-U type, Structural parameters, Geometric parameters.

\section{INTRODUCTION}

Due to the development of aerospace and aviation technology, it requires not only the high resolution but also the micromation of the size and dimension in order to realize the deflection bearing in small area. After conducting various experiments exploring different types of elastomeric bearing, people progressively developed the flexure hinge with small dimension, without mechanical friction and without gap. Flexure Hinge is widely used in gyroscope, acceleration meter, precision balance, missile control nozzle shape waveguide antenna and etc., and immediately, and it achieves unprecedented high precision and stability. In recent years, flexure hinge has been also applied in precision displacement operating stage and field of robotics [1].

Currently, in for the study of flexure hinge, the researchers focus on various performance objectives of various structure flexure hinges. Y. Tian of Tianjin University, developed $\mathrm{V}$ type flexure hinge structure, deduced the flexibility computation formula for $\mathrm{V}$ type flexure hinge, analyzed the stiffness properties and motion precision properties in different loads and along different axes, and conducted their finite-element verification [2]. Researchers such as Zhang Jing zhu and Xu Cheng studied single-axis straight circular arc flexure hinge [3]; Nicolae Lobonity studied the rotation capacity, rotation accuracy, maximum stress and strain efficiency of double axis flexure hinge, and verify its correctness by applying biaxial flexure hinge parabola [4]; Chen Guimin focused on the properties of elliptical flexure hinge to conduct the analytical computation [5, 6]; Ren Ning conducted the computation

\footnotetext{
*Address correspondence to this author at the School of Mechanical and Power Engineering North University of China Taiyuan Shanxi 030051, P.R. China; Tel: 13835113159; E-mail: s20031261jj@sina.com

Foundation item: Porject (Grant No. 51275486) supported by the National Natural Science Foundation of China
}

for measuring the stiffness of quadratic curve straight beam composite flexure hinge, and analyzed the influence of structure parameters on the stiffness [7-9]; Liu Qingling Studied the variable section flexible hinge and unilateral REC mixed flexible hinge [10, 11]; Zhou Xiaolin, etc., calculated rotation stiffness of straight circular flexible hinges, obtained parameters of flexible hinge to find how it affected the stiffness of the flexible hinge in PW full model, PW simplified model and WZ model [12]; Zhang Zhijie, etc., analyzed and calculated the secant curve shaped flexible hinge [13]; Cao Feng and Cheng Aiwu respectively analyzed and calculated biaxial elliptical and straight round flexure hinges $[14,15]$.

This article provides a like-U Type Flexure Hinge which is able to transform four different structures by changing the main structure parameters. Based on the Castigliano second theorem, applying the calculus theory, taking the eccentric angle parameters as the integration variable [5], and defining the proper intermediate parameters, the flexibility analytic computation formula has been deduces in this article that can be applied to all of these four different structures flexure hinges. After analyzed twelve flexure hinges of different structures. By applying finite-element method, it is found that the results are in good agreement with the results of analytic computation formula; thus verifying the validity of the analytic computation formula, realizing accurate design and computation for such flexure hinges, and providing the theoretical base for technical application of like-U type flexure hinge.

\section{FLEXIBILITY CALCULATION AND MODEL}

\subsection{Flexibility Model}

The formulation that follows is based on the following simplifying assumptions:(1) The flexure hinges consist of two symmetric cutouts: The flexure hinges are modeled and analyzed as small-displacement fixed-free Euler-Bernoulli 
beams subjected to bending produced by forces and moments; axial loading is also considered while shearing and torsional effects are not taken into account. (2) Flexure hinge is the linear elastic body. The model of like-U Type Flexure Hinge is shown in Fig. (1). Symmetrical curve in flexure hinge is formed by a straight line and an elliptical arc or circular arc. So for the like-U Type Flexure Hinge, " $a$ "means elliptical arc semi-major axis; " $b$ " means semiminor axis; " $W$ " means hinge's width; " $m$ " means kerf width; " $n$ " means depth of kerf line section; " $t$ " means the minimum thickness of the hinge. As shown in Fig. (2), when $a \neq b$ and $2 b>m$, it is the straight $\mathrm{U}$ elliptical arc flexure hinge, when $a \neq b$ and $2 b=m$, it is the elliptical arc U type flexure hinge; when $a=b$ and $2 b>m$, it is the straight $\mathrm{U}$ circular arc flexure hinge; when $a=b$ and $2 b=m$, it is the circular arc $U$ type flexure hinge, and in this article, all of these four structures are called like-U Type Flexure Hinge. In this paper, the straight $U$ elliptical arc flexure hinge has been selected, which has the general parameters as the object to do the mechanical analysis, as shown in Fig. (3). The Cartesian coordinate frame is utilized where the origin is located at the minimum thickness of the flexure hinge, and the $x$ and $y$ axes are in the height and longitudinal directions, respectively. In the mechanical analysis, set the upper end as the fixed end, and the lower end as the free end; in order to facilitate the integral calculus computing, conduct the infinitesimal dividing for the model which is based on the Cartesian coordinates, and apply load on $O$ point, since the displacement is generated on $O$ point. As shown in Fig. (4), segment the integral region for the divided infinitesimal, and set infinitesimal thickness as $d x$ and set eccentric angle as $\varphi$, as shown in Fig. (5). Thus, in three regions $A, B$ and $C$, there are respectively:

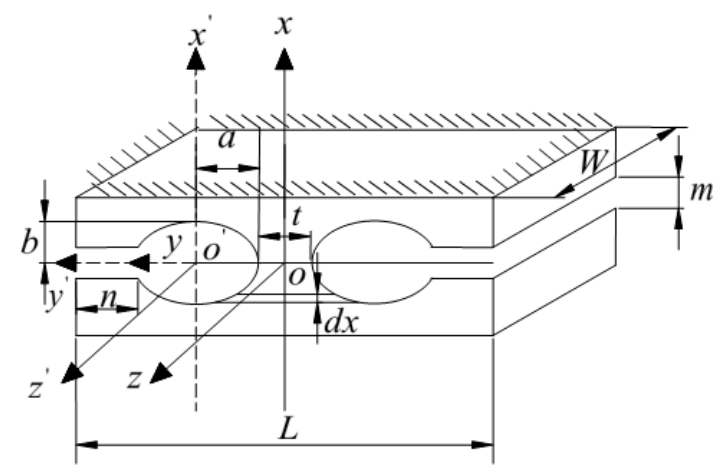

Fig. (1). The structural parameters of like-U Type flexure hinge.

$$
\begin{aligned}
& l_{A}(x)=2 a-2 a \cos \phi+t \\
& d x=b \cos \phi d \phi \quad \phi \in\left[-\frac{\pi}{2}, \frac{\pi}{2}\right] \\
& l_{B}(x)=2\left[n+a \cos \phi_{0}-a \cos \phi\right] \\
& d x=b \cos \phi d \phi \quad \phi \in\left[-\frac{\pi}{2},-\phi_{0}\right] \\
& l_{C}(x)=2\left[n+a \cos \phi_{0}-a \cos \phi\right] \\
& d x=b \cos \phi d \phi \quad \phi \in\left[\phi_{0}, \frac{\pi}{2}\right]
\end{aligned}
$$

$$
\text { Make } \quad \frac{a}{t}=p \quad s(\phi)=2 p-2 p \cos \phi+1 \quad \frac{a}{n}=q
$$$$
k(\phi)=1+q \cos \phi_{0}-q \cos \phi
$$$$
\phi_{0}=\operatorname{arctg} \frac{2 m b}{2 a \sqrt{4 b^{2}-m^{2}}} \quad m \leq 2 b
$$

then $l_{A}(x)=t \bullet s(\phi)$

$$
l_{B}(x)=l_{C}(x)=2 n \cdot k(\phi)
$$

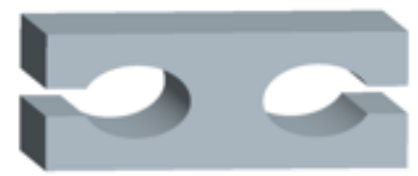

(a) $a \neq b$ and $2 b>m$

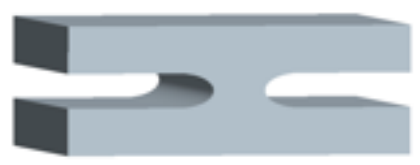

(b) $a \neq b$ and $2 b=m$

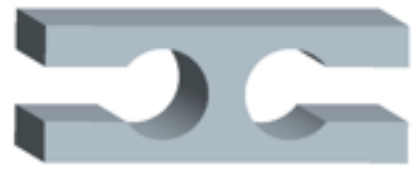

(c) $a=b$ and $2 b>m$

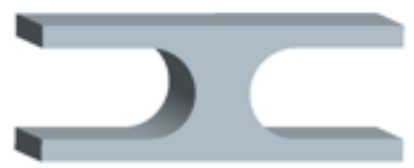

(d) $a=b$ and $2 b=m$

Fig. (2). The four different structures model of like-U Type flexure hinge.

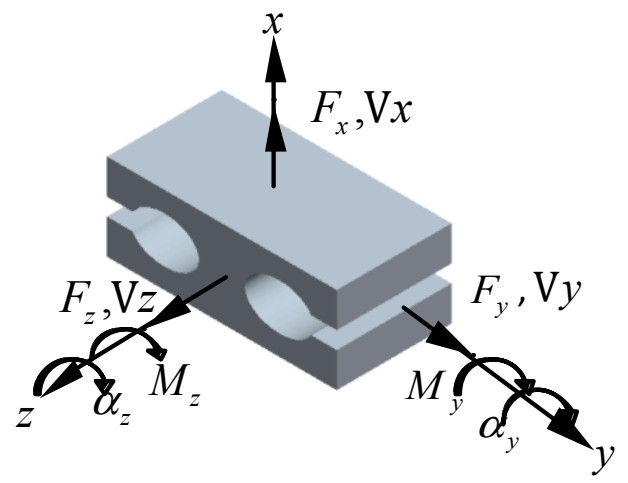

Fig. (3). The loads of like-U Type flexure hinge.

\subsection{Flexibility Computation}

In order to derive closed-form compliance equations of filleted U-shaped flexure hinges, the Castigliano's second theorem is adopted and written as follows: 
$\delta_{i}=\frac{\partial U}{\partial P_{i}}$

Where, " $U$ "is the structural distortion energy, $P_{i}$ is generalized force, $\delta_{i}$ is displacement corresponding to force, curving distortion energy is:

$U=\int_{l} \frac{M^{2} d x}{2 E I}$

tension distortion energy is

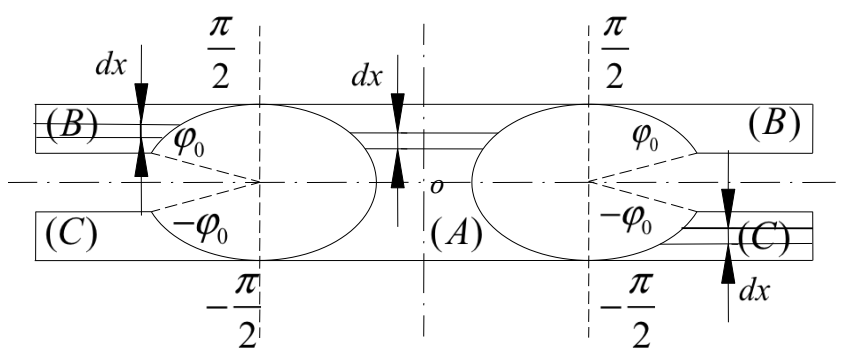

Fig. (4). The flexibility calculation partition.

$U=\int_{l} \frac{F^{2} d x}{2 E I}$

in the formula, " $E$ " is the elastic modulus of the material, and " $I$ "is the moment of inertia, then there is:

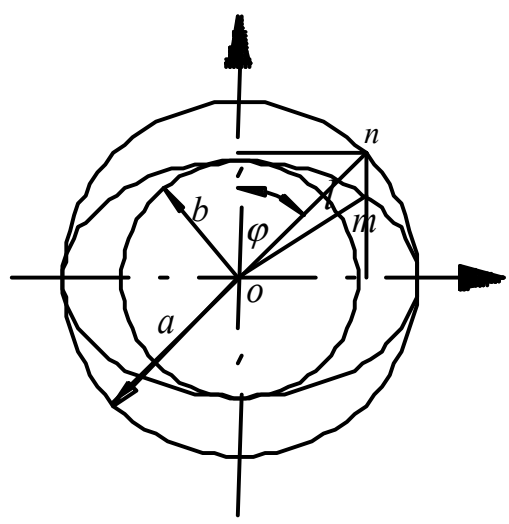

Fig. (5). Eccentric angle of an ellipse.

(1) Angular compliance of $z$-axis

The flexure hinge subject to the bending moment $M_{z}$ will rotate about $z$-axis, and the angular displacement is denoted by $\alpha_{z}$, the compliance equation is developed as follows:

$$
\begin{aligned}
& C_{\alpha_{z}-M_{z}}=\frac{\alpha_{z}}{M_{z}}=\int_{l} \frac{d x}{E I_{z}(x)} \\
& =\frac{12 b}{E W t^{3}} \int_{-\frac{\pi}{2}}^{\frac{\pi}{2}} \frac{\cos \phi}{s(\phi)^{3}} d \phi+\frac{3 b}{E W n^{3}} \int_{\phi_{0}}^{\frac{\pi}{2}} \frac{\cos \phi}{k(\phi)^{3}} d \phi \\
& =\frac{3 b}{2 E W(t p)^{3}} x_{1}+\frac{3 b}{E W(n q)^{3}} x_{2}
\end{aligned}
$$

The application of force $F_{y}$ at the free end of the flexure hinge can generate bending moment acting on the hinge; the compliance equation about $\mathrm{z}$-axis is obtained as follows:

$C_{\alpha_{z}-F_{y}}=\frac{\alpha_{z}}{F_{y}}=\int_{l} \frac{x d x}{E I_{z}(x)}$

$=\frac{12 b^{2}}{E W t^{3}} \int_{-\frac{\pi}{2}}^{\frac{\pi}{2}} \frac{\sin \phi \cos \phi+\cos \phi}{s(\phi)^{3}} d \phi$

$+\frac{3 b^{2}}{2 E W n^{3}}\left[\int_{-\frac{\pi}{2}}^{-\phi_{0}} \frac{\sin \phi \cos \phi+\cos \phi}{k(\phi)^{3}} d \phi\right.$

$\left.+\int_{\phi_{0}}^{\frac{\pi}{2}} \frac{\sin \phi \cos \phi+\cos \phi}{k(\phi)^{3}} d \phi\right]$

$=\frac{12 b^{2}}{E W t^{3}} \int_{-\frac{\pi}{2}}^{\frac{\pi}{2}} \frac{\cos \phi}{s(\phi)^{3}} d \phi+\frac{3 b^{2}}{E W n^{3}} \int_{\phi_{0}}^{\frac{\pi}{2}} \frac{\cos \phi}{k(\phi)^{3}} d \phi$

$=\frac{3 b^{2}}{2 E W(t p)^{3}} x_{1}+\frac{3 b^{2}}{8 E W(n q)^{3}} x_{2}$

Among them:

$x_{1}=\frac{6 s^{2} a_{2}+\left(2 s^{2}+1\right) e}{e^{5} \cdot s} \quad s=\frac{1+2 p}{2 p}$

$x_{2}=\frac{\sqrt{6}}{2} f k^{2} a_{0}-4 l^{3} k f^{2}+4 k^{3} f^{2} l^{4}-2 k^{2} l f^{2}$

$+6 f k^{4} a_{0}-12 f k^{3} a_{0}+f l^{4}-2 f^{2} l^{2}-2 k f^{2}$

$+12 k^{3} f a_{1}-6 f^{3} a_{1}+3 k^{2} f^{2}-4 k^{3} f^{2}+4 l k f^{2}$

$+2 k^{4} f^{2} l^{4}+4 f^{2} k^{4} l^{2}+6 f k^{4} l^{4} a_{0}+12 f k^{4} l^{2} a_{0}$

$+12 f k^{3} l^{4} a_{0}+6 f k^{2} l^{4} a_{0}-12 f k^{2} l^{2} a_{0}-2 f^{2} k^{2} l^{2}$

$+3 f^{2} k^{2} l^{4}+2 f^{2} k l^{4}-4 f^{2} k^{4} l^{3}-4 f^{2} k^{4} l$

$+2 f^{2} k^{3} l-2 f^{2} k^{2} l^{3}-12 f k^{4} l a_{1}-6 f k^{4} l^{4} a_{1}$

$-12 f k^{3} l^{4} a_{1}+12 f k^{2} l^{2} a_{1}-6 f k^{2} l^{4} a_{1}-2 f^{2} k^{3} l^{3}$

$+\frac{2 k^{4} f^{2}}{f^{10} k\left[k+k l^{2}+l^{2}-1\right]^{2}}$

$f=\sqrt{k^{2}-1} \quad l=\operatorname{tg}\left(1 / 2 \phi_{0}\right) \quad k=\left(1+q \cos \phi_{0}\right) / q$

$a_{0}=\operatorname{arctg} \sqrt{\frac{k+1}{k-1}} \quad a_{1}=\operatorname{arctg}\left[\sqrt{\frac{k+1}{k-1}} \operatorname{tg}\left(\frac{1}{2} \phi_{0}\right)\right]$

(2) Angular compliance about $y$-axis

The angular displacement of the " $y$ " axis is generated by two parts of load; the angular displacement generated by moment " $M_{y}$ "; and the angular displacement generated by force " $F_{z}$ ", their flexibilities are:

$$
\begin{aligned}
& C_{\alpha_{y}-M_{y}}=\frac{\alpha_{y}}{M_{y}}=\int_{l} \frac{d x}{E I_{y}(x)}(6) \\
& =\frac{12 b}{E W^{3} t} \int_{-\frac{\pi}{2}}^{\frac{\pi}{2}} \frac{\cos \phi}{s(\phi)} d \phi+\frac{6 b}{E W^{3} n}\left[\int_{-\frac{\pi}{2}}^{-\phi_{0}} \frac{\cos \phi}{k(\phi)} d \phi\right. \\
& \left.+\int_{\phi_{0}}^{\frac{\pi}{2}} \frac{\cos \phi}{k(\phi)} d \phi\right]=\frac{12 b}{E W^{3} t} \int_{-\frac{\pi}{2}}^{\frac{\pi}{2}} \frac{\cos \phi}{s(\phi)} d \phi
\end{aligned}
$$


$+\frac{12 b}{E W^{3} n} \int_{\phi_{0}}^{\frac{\pi}{2}} \frac{\cos \phi}{k(\phi)} d \phi$

$=\frac{6 b}{E t p W^{3}} x_{3}+\frac{12 b}{E n q W^{3}} x_{4}$

$C_{\alpha_{y}-F_{z}}=\frac{\alpha_{y}}{F_{z}}=\int_{l} \frac{x d x}{E I_{y}(x)}$

$=\frac{12 b^{2}}{E W^{3} t} \int_{-\frac{\pi}{2}}^{\frac{\pi}{2}} \frac{\sin \phi \cos \phi+\cos \phi}{s(\phi)} d \phi$

$+\frac{6 b^{2}}{E W^{3} n}\left[\int_{-\frac{\pi}{2}}^{-\phi_{0}} \frac{\sin \phi \cos \phi+\cos \phi}{k(\phi)} d \phi\right.$

$\left.+\int_{\phi_{0}}^{\frac{\pi}{2}} \frac{\sin \phi \cos \phi+\cos \phi}{k(\phi)} d \phi\right]$

$=\frac{12 b^{2}}{E W^{3} t} \int_{-\frac{\pi}{2}}^{\frac{\pi}{2}} \frac{\cos \phi}{s(\phi)} d \phi$

$+\frac{6 b^{2}}{E W^{3} n}\left[\int_{-\frac{\pi}{2}}^{-\phi_{0}} \frac{\sin \phi \cos \phi+\cos \phi}{k(\phi)} d \phi\right.$

$\left.+\int_{\phi_{0}}^{\frac{\pi}{2}} \frac{\sin \phi \cos \phi+\cos \phi}{k(\phi)} d \phi\right]$

$=\frac{12 b^{2}}{E W^{3} t} \int_{-\frac{\pi}{2}}^{\frac{\pi}{2}} \frac{\cos \phi}{s(\phi)} d \phi+\frac{12 b^{2}}{E W^{3} n} \int_{\phi_{0}}^{\frac{\pi}{2}} \frac{\cos \phi}{k(\phi)} d \phi$

$=\frac{6 b^{2}}{E t p W^{3}} x_{3}+\frac{12 b^{2}}{E n q W^{3}} x_{4}$

Among them:

$x_{3}=\frac{4 s a_{2}-\pi e}{e} \quad e=\sqrt{s^{2}-1}$

$x_{4}=\left[2 f k a_{0}-\frac{1}{2} \pi f^{2}-2 k f a_{1}+\phi_{0} f^{2}\right] / f^{2}$

(3) Linear compliance along $Z$-axis

The bending moment $M_{y}$ and force $F_{z}$ can also result in the linear displacement of the flexure hinge along $z$-axis. The linear compliances along $\mathrm{z}$-axis under the bending moment $M_{y}$ and force $F_{z}$ are respectively given as follows:

$C_{z-F_{z}}=\frac{\Delta z}{F_{z}}=\int_{l} \frac{x^{2} d x}{E I_{y}(x)}$

$=\frac{12 b^{3}}{E W^{3} t} \int_{-\frac{\pi}{2}}^{\frac{\pi}{2}} \frac{2 \sin \phi \cos \phi+\cos \phi+\sin ^{2} \phi \cos \phi}{l(\phi)} d \phi$

$+\frac{6 b^{3}}{E W^{3} n}\left[\int_{-\frac{\pi}{2}}^{-\phi_{0}} \frac{2 \sin \phi \cos \phi+\cos \phi+\sin ^{2} \phi \cos \phi}{k(\phi)} d \phi\right.$

$\left.+\int_{\phi_{0}}^{\frac{\pi}{2}} \frac{2 \sin \phi \cos \phi+\cos \phi+\sin ^{2} \phi \cos \phi}{k(\phi)} d \phi\right]$

$=\frac{12 b^{3}}{E W^{3} t} \int_{-\frac{\pi}{2}}^{\frac{\pi}{2}} \frac{\cos \phi+\sin ^{2} \phi \cos \phi}{l(\phi)} d \phi$

$+\frac{12 b^{3}}{E W^{3} n} \int_{\phi_{0}}^{\frac{\pi}{2}} \frac{\cos \phi+\sin ^{2} \phi \cos \phi}{k(\phi)} d \phi$

$=\frac{1}{p} x_{3}-\frac{1}{2 p} x_{7}+\frac{2}{q} x_{4}-\frac{1}{q} x_{8}$
Among them:

$x_{7}=\left[4 s^{3} a_{2}-\pi e s^{2}-2 s e-\frac{1}{2} \pi e\right] / e$

$x_{8}=-\frac{1}{4}\left[-2 \phi_{0} f^{2}+4 \pi f^{2} k^{2} l^{2}+2 \pi f^{2} k^{2} l^{4}\right.$

$+4 f^{2} l^{3}-4 f^{2} l+16 f k^{3} l^{2} h+2 \pi f^{2} l^{2}$

$+\pi f^{2} l^{4}+8 f k^{3} l^{4} h-8 f^{2} k^{2} l^{4} \operatorname{arctg} l$

$-8 f^{2} k l-8 f^{2} k^{2} \operatorname{arctg} l+2 \pi f^{2} k^{2}$

$-16 f k^{3} l^{2} g-8 f k^{3} l^{4} g-8 f^{2} k l^{3}$

$+4 f^{2} k+8 f^{2} k l^{2}-2 \phi_{0} f^{2} l^{4}$

$\left.+4 f^{2} k l^{4}-4 \phi_{0} f^{2} l^{2}\right] / f^{2}\left(1+l^{2}\right)^{2}$

$e=\sqrt{s^{2}-1} \quad a_{2}=\operatorname{arctg} \sqrt{\frac{s+1}{s-1}}$

The linear distortion " $\Delta z$ " along “ $z$ " axis generated by moment " $M_{y}$ ", the flexibility expression is

$C_{z-M_{y}}=\frac{\Delta z}{F_{z}}$

According to reciprocal theorem, there is:

$C_{z-M_{y}}=C_{\alpha_{y}-F_{z}}=\frac{12 b^{2}}{E W^{3} t} \int_{-\frac{\pi}{2}}^{\frac{\pi}{2}} \frac{\cos \phi}{s(\phi)} d \phi$

$+\frac{12 b^{2}}{E W^{3} n} \int_{\phi_{0}}^{\frac{\pi}{2}} \frac{\cos \phi}{k(\phi)} d \phi=\frac{6 b^{2}}{E t p W^{3}} x_{3}+\frac{12 b^{2}}{E n q W^{3}} x_{4}$

(4) Linear compliance along $y$-axis

The flexure hinge can generate linear displacement along y-axis due to the bending moment $M_{z}$. and force $F_{y}$, The linear compliances along $y$-axis are respectively given as follows:

$C_{y-F_{y}}=\frac{\Delta y}{F_{y}}$

$=\frac{12 b^{3}}{E W t^{3}} \int_{-\frac{\pi}{2}}^{\frac{\pi}{2}} \frac{2 \sin \phi \cos \phi+\cos \phi+\sin ^{2} \phi \cos \phi}{s(\phi)^{3}} d \phi$

$=\frac{12 b^{3}}{E W t^{3}} \int_{-\frac{\pi}{2}}^{\frac{\pi}{2}} \frac{\cos \phi+\sin ^{2} \phi \cos \phi}{s(\phi)^{3}} d \phi$

$+\frac{3 b^{3}}{E W n^{3}} \int_{\phi_{0}}^{\frac{\pi}{2}} \frac{\cos \phi+\sin ^{2} \phi \cos \phi}{k(\phi)^{3}} d \phi$

$=\frac{12 b^{3}}{E W t^{3}}\left(\frac{1}{4 p^{3}} x_{1}-\frac{1}{8 p^{3}} x_{5}\right)+\frac{3 b^{3}}{E W n^{3}}\left(\frac{2}{q^{3}} x_{2}-\frac{1}{q^{3}} x_{6}\right)$

Among them:

$x_{5}=\left[2 s h+2 \pi e s^{2}-\pi e+5 s e-\pi e s^{4}-10 s^{3}\right.$

$\left.+4 s^{5} h-2 l^{3} e\right] / e^{5}$

$x_{6}=-\frac{1}{2}\left(-2 \phi_{0} f^{2}-12 f k a_{1}-8 k^{6} a_{0} f-6 k^{5} a_{0} f\right.$

$+20 k^{4} a_{0} f+2 k^{3} a_{0} f-6 f^{2} \pi k^{4} l^{2}-4 f^{2} \pi k^{3} l^{4}$

$-f^{2} \pi k^{2} l^{4}+6 f^{2} \pi k^{2} l^{2}+2 f^{2} \pi k l^{4}-f^{2} \pi k^{4} l^{4}$ 

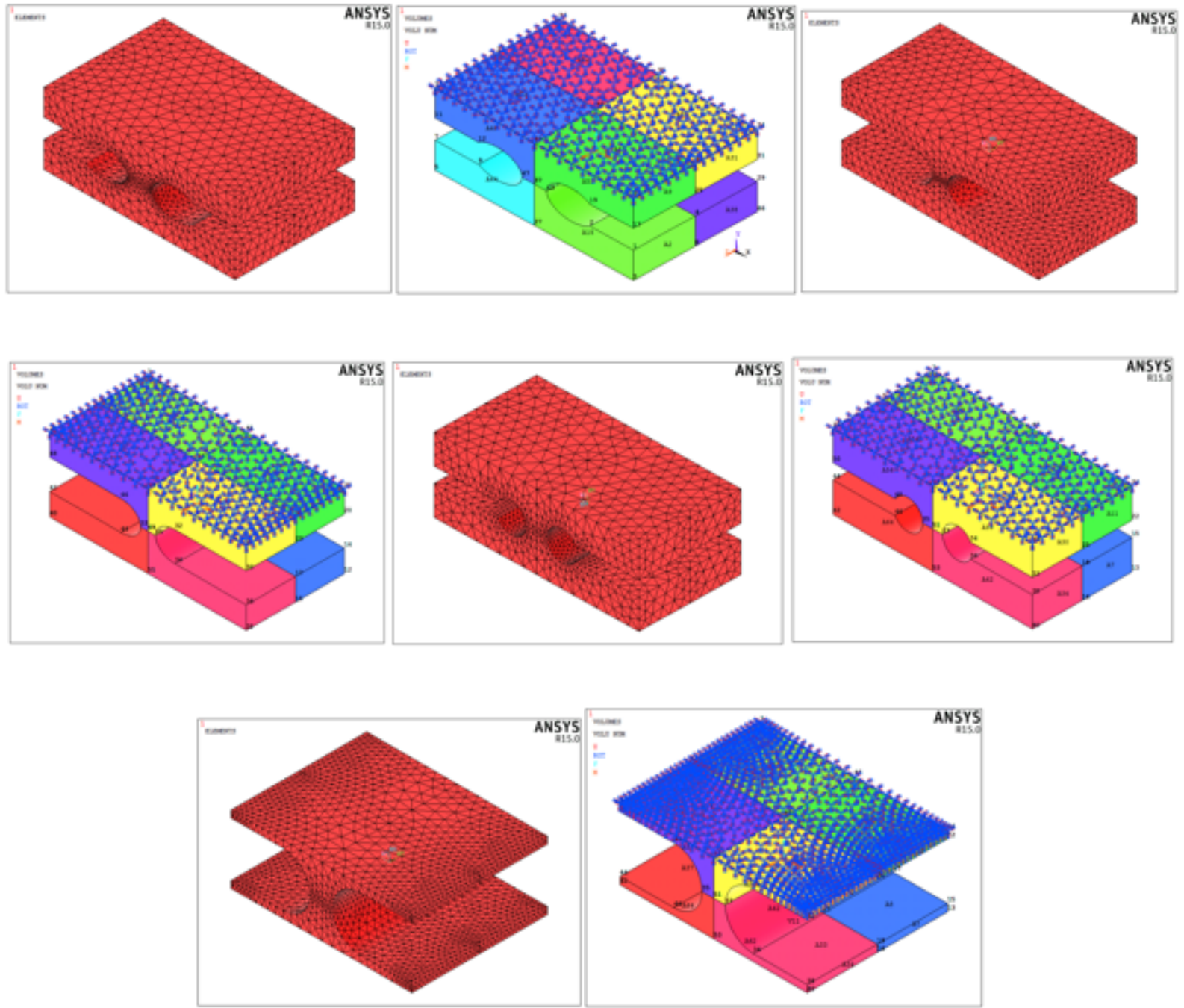

Fig. (6). Finite element model and boundary conditions of flexure hinge.

Table 1. Physical parameters and geometric parameters of flexure hinges.

\begin{tabular}{|c|c|c|c|c|c|}
\hline Number & $2 \mathrm{a} / \mathrm{mm}$ & $2 \mathrm{~b} / \mathrm{mm}$ & $\mathbf{m} / \mathbf{m m}$ & $\mathbf{W} / \mathbf{m m}$ & $\mathrm{t} / \mathrm{mm}$ \\
\hline 1 & 12 & 8 & 3 & 20 & 5 \\
\hline 2 & 10 & 6 & 4 & 25 & 5 \\
\hline 3 & 14 & 10 & 6 & 30 & 3 \\
\hline 4 & 8 & 6 & 6 & 20 & 3 \\
\hline 5 & 12 & 8 & 8 & 25 & 4 \\
\hline 6 & 14 & 8 & 8 & 30 & 3 \\
\hline 7 & 6 & 6 & 3 & 20 & 2 \\
\hline 8 & 10 & 10 & 6 & 25 & 4 \\
\hline 9 & 12 & 12 & 8 & 30 & 3 \\
\hline 10 & 8 & 8 & 8 & 20 & 3 \\
\hline 11 & 10 & 10 & 10 & 25 & 4 \\
\hline 12 & 12 & 12 & 12 & 30 & 5 \\
\hline
\end{tabular}


Table 2. Comparison between finite element and analytical results for the compliance factors.

\begin{tabular}{|c|c|c|c|c|c|c|}
\hline \multirow{2}{*}{ Flexibility } & \multicolumn{3}{|c|}{ Linear Mixed Elliptical arc Flexure Hinges } & \multicolumn{3}{|c|}{ Elliptical arc U-Type Flexure Hinges } \\
\hline & Analytic & FEM & Error (\%) & Analytic & FEM & Error (\%) \\
\hline \multirow{3}{*}{$C_{\alpha_{z}-\mu_{z}} \times 10^{-12}$} & 23.55 & 24.04 & 2.08 & 9.244 & 10.01 & 8.329 \\
\hline & 58.28 & 51.1 & 7.18 & 3.17 & 3.211 & 1.29 \\
\hline & 5.115 & 4.91 & 4 & 4.072 & 3.986 & 2.11 \\
\hline \multirow{3}{*}{$C_{\alpha_{z}-F_{y}} \times 10^{-12}$} & 38.96 & 38.89 & 1.79 & 24.49 & 22.79 & 1.33 \\
\hline & 17.08 & 17.69 & 3.571 & 5.178 & 5.083 & 1.83 \\
\hline & 3.352 & 3.421 & 2.06 & 2.135 & 2.133 & 0.2 \\
\hline \multirow{3}{*}{$C_{\alpha_{y}-F_{z}} \times 10^{-12}$} & 33.43 & 31.68 & 5.23 & 14.21 & 14.76 & 3.871 \\
\hline & 12.79 & 12.81 & 0.16 & 9.06 & 9.048 & 0.132 \\
\hline & 6.908 & 6.743 & 2.39 & 4.523 & 4.641 & 2.405 \\
\hline \multirow{3}{*}{$C_{\alpha_{y}-M_{y}} \times 10^{-12}$} & 8.356 & 7.764 & 7.085 & 4.736 & 4.812 & 1.605 \\
\hline & 4.363 & 4.415 & 1.183 & 2.265 & 2.247 & 0.795 \\
\hline & 1.382 & 1.295 & 6.295 & 1.131 & 1.348 & 2.645 \\
\hline \multirow{3}{*}{$C_{x-F_{x}} \times 10^{-11}$} & 27.86 & 26.65 & 4.343 & 15.79 & 15.72 & 0.443 \\
\hline & 22.21 & 22.76 & 2.495 & 11.8 & 12.13 & 2.797 \\
\hline & 10.36 & 10.44 & 0.772 & 8.48 & 8.454 & 0.307 \\
\hline \multirow{3}{*}{$C_{y-F_{y}} \times 10^{-11}$} & 58.12 & 58.67 & 0.946 & 8.109 & 8.202 & 1.148 \\
\hline & 33.9 & 34.58 & 2.005 & 8.24 & 8.035 & 2.448 \\
\hline & 25.48 & 24.06 & 5.572 & 12.98 & 12.88 & 0.77 \\
\hline \multirow{3}{*}{$C_{z-F_{z}} \times 10^{-12}$} & 41.03 & 40.25 & 1.901 & 4.236 & 4.158 & 1.841 \\
\hline & 10.8 & 10.99 & 1.759 & 12.99 & 12.57 & 3.233 \\
\hline & 20.16 & 20.62 & 2.298 & 6.336 & 6.348 & 0.189 \\
\hline \multirow{2}{*}{ Flexibility } & \multicolumn{3}{|c|}{ Linear Mixed arc Flexure Hinges } & \multicolumn{3}{|c|}{ Arc U-Type Flexure Hinges } \\
\hline & Analytic & FEM & Error (\%) & Analytic & FEM & Error $(\%)$ \\
\hline \multirow{3}{*}{$C_{\alpha_{z}-\mu_{z}} \times 10^{-12}$} & 8.5 & 8.476 & 0.282 & 11.07 & 11.26 & 1.716 \\
\hline & 11.23 & 11.45 & 1.959 & 9.866 & 9.669 & 2.831 \\
\hline & 2.573 & 2.398 & 6.801 & 8.448 & 8.505 & 0.675 \\
\hline \multirow{3}{*}{$C_{\alpha_{z}-F_{y}} \times 10^{-12}$} & 23.39 & 23.46 & 0.727 & 42.93 & 41.88 & 2.443 \\
\hline & 48.02 & 47.16 & 1.791 & 47.11 & 48.05 & 1.995 \\
\hline & 2.871 & 2.968 & 3.383 & 47.12 & 47.48 & 0.764 \\
\hline \multirow{3}{*}{$C_{\alpha_{y}-F_{z}} \times 10^{-12}$} & 13.59 & 12.82 & 5.666 & 21.2 & 20.79 & 1.943 \\
\hline & 19.63 & 19.08 & 2.801 & 16.56 & 15.78 & 4.71 \\
\hline & 8.424 & 8.391 & 0.392 & 17.03 & 17.62 & 3.464 \\
\hline \multirow{3}{*}{$C_{\alpha_{y}-M_{y}} \times 10^{-12}$} & 4.953 & 4.885 & 1.373 & 5.299 & 5.257 & 2.793 \\
\hline & 3.926 & 3.921 & 1,274 & 3.311 & 3.332 & 0.634 \\
\hline & 1.404 & 1.401 & 0.214 & 2.823 & 2.829 & 1.317 \\
\hline \multirow{3}{*}{$C_{x-F_{x}} \times 10^{-11}$} & 16.51 & 16.08 & 2.604 & 17.66 & 16.83 & 4.7 \\
\hline & 20.45 & 20.81 & 1.174 & 17.25 & 17.48 & 1.333 \\
\hline & 10.53 & 10.61 & 0.754 & 21.28 & 20.79 & 2.303 \\
\hline \multirow{3}{*}{$C_{y-F_{y}} \times 10^{-11}$} & 6.972 & 6.811 & 2.309 & 19.44 & 19.87 & 2.212 \\
\hline & 29.91 & 29.76 & 0.502 & 27.31 & 27.63 & 1.172 \\
\hline & 13.73 & 12.88 & 6.191 & 38.89 & 37.58 & 3.368 \\
\hline \multirow{3}{*}{$C_{z-F_{z}} \times 10^{-12}$} & 13.82 & 13.66 & 1.158 & 29.31 & 28.84 & 1.604 \\
\hline & 33.23 & 34.58 & 4.063 & 35.11 & 35.62 & 1.453 \\
\hline & 20.35 & 19.92 & 2.13 & 27.94 & 26.07 & 6.693 \\
\hline
\end{tabular}


$-3 f^{2} \pi k^{2} l^{4}-12 f^{2} k^{2} l-5 f^{2} k+10 f^{2} k^{2}-3 f^{2} k^{3}$

$-12 \phi_{0} f^{2} k^{2} l^{2}+2 \phi_{0} f^{2} k^{2} l^{2}-4 \phi_{0} f^{2} k l^{4}$

$+12 \phi_{0} f^{2} k^{4} l^{2}-4 \phi_{0} f^{2} k^{6} l^{2}-2 \phi_{0} f^{2} k^{6} l^{4}$

$-4 \phi_{0} f^{2} k^{5} l^{4}+2 \phi_{0} f^{2} k^{5} l^{4}+8 \phi_{0} f^{2} k^{3} l^{4}$

$+\pi f^{2} k^{6} l^{4}+2 \pi f^{2} l^{2}+2 \pi f^{2} k^{5} l^{4}$

$+6 f k^{5} l^{4} a_{1}+2 f^{2} k^{5}+20 f k^{4} l^{4} a_{1}$

$-2 f k^{3} l^{4} a_{1}-44 f k^{3} l^{2} a_{1}-8 f k^{6} l^{4} a_{1}$

$-24 f k^{2} l^{4} a_{1}-12 f l^{4} a_{1}+24 f k l^{2} a_{1}$

$-14 f^{2} k^{3} l^{2}+10 f^{2} k l^{2}+\pi f^{2} k^{6}-2 \pi f^{2} k^{5}$

$+4 \pi f^{2} k^{3}+6 f k^{5} a_{1}-\pi f^{2} k-2 \pi f^{2} k$

$-24 f k l^{2} a_{0}+12 f k l^{4} a_{0}-8 \phi_{0} f^{2} k^{3}-2 \phi_{0} f^{2} l^{4}$

$+2 f k^{3} l^{4} a_{0}+44 f k^{3} l^{2} a_{0}+2 f^{2} k^{4} l^{4}+4 f^{2} k^{5} l^{2}$

$-4 f k^{7} l^{4}-8 f k^{4} l^{2} a_{1}+28 f^{2} k^{5} l^{2} a_{1}+4 \phi_{0} f^{2} l^{2}$

$-4 f^{2} k^{4}-\pi f^{2} k^{4}-4 f^{2} k^{5} l+24 f k^{2} l^{4} a_{0}$

$-4 f^{2} k^{5} l^{3}+4 f k^{7} l^{4} a_{0}+8 f k^{7} l^{4} a_{0}+8 f k^{7} l^{2} a_{0}$

$\left.+8 f k^{6} l^{4} a_{0}-6 f k^{5} l^{4} a_{0}-28 f k^{5} l^{2} a_{0}-2 f k^{3} a_{1}\right)$

$l f^{2}\left(k+k l^{2}+l^{2}-1\right)^{2}(k+1)^{2}$

$f=\sqrt{k^{2}-1} \quad l=\operatorname{tg}\left(\frac{1}{2} \phi_{0}\right) \quad k=\frac{1+q \cos \phi_{0}}{q}$

$a_{0}=\operatorname{arctg} \sqrt{\frac{k+1}{k-1}} \quad a_{1}=\operatorname{arctg}\left[\sqrt{\frac{k+1}{k-1}} \operatorname{tg}\left(\frac{1}{2} \phi_{0}\right)\right]$

For the linear distortion " $\Delta y$ ” generated by moment “ $M_{z}$ ", the flexibility expression is:

$C_{\Delta y-M_{z}}=\frac{\Delta y}{M_{z}}$

on the basis of reciprocal theorem, there is:

$C_{y-M_{z}}=C_{\alpha_{z}-F_{y}}=\frac{12 b^{2}}{E W t^{3}} \int_{-\frac{\pi}{2}}^{\frac{\pi}{2}} \frac{\cos \phi}{s(\phi)^{3}} d \phi$

$+\frac{3 b^{2}}{E W n^{3}} \int_{\phi_{0}}^{\frac{\pi}{2}} \frac{\cos \phi}{k(\phi)^{3}} d \phi=\frac{3 b^{2}}{2 E W(t p)^{3}} x_{1}+\frac{3 b^{2}}{8 E W(n q)^{3}} x_{2}$

\section{(5) Linear compliance along $x$-axis}

The linear distortion " $\Delta x$ " generated by force $F_{x}$, the linear compliances along $x$-axis are given as follows:

$$
\begin{aligned}
& C_{x-F_{x}}=\frac{\Delta x}{F_{x}} \\
& =\frac{b}{E W t} \int_{-\frac{\pi}{2}}^{\frac{\pi}{2}} \frac{\cos \phi}{s(\phi)} d \phi+\frac{b}{2 E W n}\left[\int_{-\frac{\pi}{2}}^{-\phi_{0}} \frac{\cos \phi}{s(\phi)} d \phi\right.
\end{aligned}
$$

\section{REFERENCES}

Yu Jing-jun, Pei Chao, Bi Shu-shen, Zong Guang-hua, Zhang Xian-ming. Design method advances of flexible hinge mechanism [J]. Journal of Mechanical Engineering, 2010, 46(13): 2-12.

$$
\begin{aligned}
& \left.+\int_{\phi_{0}}^{\frac{\pi}{2}} \frac{\cos \phi}{k(\phi)} d \phi\right]=\frac{b}{E W t} \int_{-\frac{\pi}{2}}^{\frac{\pi}{2}} \frac{\cos \phi}{s(\phi)} d \phi+\frac{b}{E W n} \int_{\phi_{0}}^{\frac{\pi}{2}} \frac{\cos \phi}{k(\phi)} d \phi \\
& =\frac{b}{2 E t p W} x_{3}+\frac{b}{E n q W} x_{4}
\end{aligned}
$$

\section{FINITE ELEMENT SIMULATION AND ANALYSIS}

In order to verify the flexibility formula, for the four structures Like-U type flexure hinges, we selected three groups of total twelve models to conduct the model analysis, fixed the upper end, and apply unit load on center of symmetry "O" point:

$F_{x}=F_{y}=F_{z}=10^{-3} \mathrm{~N}$,

$M_{x}=M_{y}=M_{z}=10^{-6} N * m$,

$E=210 \times 10^{6} \mathrm{kpa}$, the models of four types flexure hinge finite-element analysis are shown in Fig. (6). The Physical parameters and geometric parameters of flexure hinges is shown in Table 1. The contrast results of finite element analysis and analytical solution is shown in Table 2 . It can be seen that the results of the finite element analysis and closedform equations are in good agreement with the maximum deviation being less than $9 \%$.

\section{CONCLUSION}

This article develops a like-U Type Flexure Hinge, which is able to transform four different structures by changing the structure parameters, and deduces the flexibility analytic computation formula that can be applied to all of these four different structures flexure hinges. The process of formula deducing and computing is very intricate, but the result is simplified by defining the intermediate parameters. The maximum deviation between analytic computation formula result and finite-element analysis result is less than $9 \%$, which proves the validity and reasonability of the deduced computation formula and provides the theoretical basis for technical application of like-U type flexure hinge.

\section{CONFLICT OF INTEREST}

The author confirms that this article content has no conflict of interest.

\section{ACKNOWLEDGEMENTS}

This research is sponsored by the National Natural Science Foundation of China (Grant No. 51275486).

Y. Tian, B. Shirinzadeh, D. Zhang. Closed-form compliance equations of filleted V-shaped flexure hinges for compliant mechanism design[J]. Precision Engineering, 2010, 34(3): 408418. 
[3] ZHANG Jing-zhu, XU Cheng, ZHANG Yan-jun. Flexibility computing of new flexible hinge[J]. Engineering Mechanics, 2008, 25(11): 27-32

[4] Nicolae Lobonit, Ephrahim Garcia. Two-axis flexure hinges with axially-collocated and symmetric notches[J]. Computers \& Structures, 2003, 81(13): 1329-1341.

[5] Cheng Gui-ming, Han Qi. Deep notch elliptical flexure hinges [J]. Optics and Precision Engineering, 2009, 17(3): 570-575.

[6] Cheng Gui-ming, Liu Xiao-yuan, Jia Jian-yuan. Flexibility computing of elliptical flexible hinge [J]. Chinese Journal of Mechanical Engineering, 2006, 42(Supplement): 111-115.

[7] Ren Ning, Ou Kai-liang, Wang Chang-lu, Zhou Feng. Stiffness calculation and analysis of Parabola straight beam composite flexible hinge [J]. Mechanical Science and Technology for Aerospace Engineering, 2012, 31(8): 1280-1284.

[8] Ren Ning, Ou Kai-liang, Wang Chang-lu, Zhou Feng. Stiffness calculation and analysis of Ellipse straight beam composite flexible hinge [J]. Mechine Design and Research, 2011, 27(6):27-33.

[9] Ren Ning, Ou Kai-liang, Wang Chang-lu, Zhou Feng. Stiffness research of new hyperbolic straight beam composite flexible hinge [J]. Journal of Mechanical Strength, 2012, 34(5):781-785.
[10] Liu Qing-ling. Deformation analysis and calculation method of Variable section flexible hinge [J]. Journal of Mechanical Design, 2010, 27(4):14-16.

[11] Liu Qing-ling, Wen Hai-shan, Qiu Li-fang. Flexibility calculation and performance analysis of new unilateral RCE mixed flexure hinges [J]. Engineering Mechanics, 2010, 27(10):52-56.

[12] Zhou Xiao-ling, Cui Chang-cai, Fan Wei, Fu Shi-wei, Ye Rui-fang. Calculation and analysis of three kinds of flexible hinges model [J]. Journal of Mechanical Design, 2011, 28(5):5-9.

[13] Zhang Zhi-jie, Yuan Yi-bao Calculation and analysis of Secant curvilinear flexible hinges [J]. Engineering Mechanics, 2008, 25(2):88-92.

[14] Cao Feng, Jiao Zong-xia. Design and Calculation of biaxial elliptical flexible hinges [J]. Engineering Mechanics, 2007, 24(4):178-182.

[15] Chen Ai-wu, Cao Feng, Hou Wen. The Flexbility calculation of biaxial right circular flexible hinges[J]. Journal of Basic Science and Engineering, 2010, 18(5):838-846.

This is an open access article licensed under the terms of the (https://creativecommons.org/licenses/by/4.0/legalcode), which permits unrestricted, non-commercial use, distribution and reproduction in any medium, provided the work is properly cited. 